\title{
Slotted ALOHA CDMA By Dual Model Path Gains
}

\author{
Hoga Saragih', \\ Djoko Soetarno ${ }^{2}$
}

E-mail: hogasaragih@gmail.com,djokosutarno@yahoo.com

Diterima: 11 Agustus 2009 / Disetujui: 31 Agusutus 2009

\begin{abstract}
Throughput Slotted ALOHA CDMA by dual model path gain Nakagami/Nakagami fading channel by capture effect is analyzed. The approach of fading effect to BER is calculated by path gain. Path gain for desired signal and interference signal is unequal. Therefore, at this research BER used on S-ALOHA CDMA is BER with two different model path gains at Nakagami/ Nakagami fading channel with capture effect. Result of calculation indicates that throughput of slotted ALOHA CDMA are getting better with increase level of parameter fading signal and long code. Others throughput slotted ALOHA CDMA also better progressively with the decrease of capture ratio and simultaneous user.
\end{abstract}

keywords: Slotted ALOHA (s-aloha), CDMA, s-aloha cdma, Nakagami fading, CAPTURE EFFECT

\section{Introduction}

Wireless communication based on code-division multiple accesses (CDMA) have a capability to handling multiple access transmission, overcoming multipath fading and cochannel interference, and also enhancing capacity of the system. [1]. Multipath fading causes the quality of services get decreasing while cochannel interference causes low capacity of system. There are two kinds of parameter that usually used for showing performance of CDMA's system are outage probability and bit error rate (BER) [2]. BER calculation for CDMA has been used by researchers to calculate in a variety of approximation where they use standard Gaussian approximation (SGA) often. The results of calculation have been implementing in variety of channel fading cases [3]-[7].

In SGA, central limit theorem (CLT) is used, then we assume that signal effect of multiple access interference (MAI) with additive white-Gaussian process, variance of MAI is used to calculate signal-to-interference ratio (SIR). SGA is an easy approximation to

1. Dosen Magister Teknologi Informasi Universitas Bina Nusantara

Jl. Kebun Jeruk Raya No. 27, Jakarta Barat 11530

2. Dosen Magister Sistem Informasi Universitas Bina Nusantara

Jl. Kebun Jeruk Raya No. 27, Jakarta Barat 11530 
implement because it uses the rate of SNR directly but the analysis of performance is very optimistic if the amount of users is small especially [3].

BER's calculation for CDMA based on CLT, it used for analysis CDMA system in the fading channel with Rayleigh distribution and synchronizing random sequences or asynchronizes [7]. The influencing of amount of users is effect on BER when we measured in a certain SNR. In [8], influencing of channel coding and diversity to BER's estimation in Rayleigh channel has been explaining specifically. The combination of spread spectrum modulation with channel coding and diversity is able to gain a high performance in one of application in this technique

The calculation of BER's cellular system in Rayleigh/Rician channel has been analyzed [9]. In Rayleigh/Rician channel, desired signal modeled in Rayleigh distribution, while interfering signals modeled in Rician Lee distribution, [10] has been explaining about the influence of channel coding and diversity order toward BER's CDMA in Rician channel, while Sasamori and his friends [11] have been generate BER's CDMA in Rician channel.

The influencing of cochannel interference that go through fading with Nakagami distribution has been explain in [2],[12]. In [2], expression of closed form from BER system for mobile communication to generate binary phase shift keying (BPSK) and binary frequency shift keying (BFSK). While in [12], the influence of cochannel interference with Nakagami model is compare with Rician/Rayleigh model. In CDMA system, the influence of fading to BER is get from determine SIR first then path gain through assumed it has same rate neither main signal or interfering signal.

The analysis of ALOHA perfomance in fading channel has been explain before [13]. The analysis of S-ALOHA perfomance in channel fading Rayleigh has been explain [14],[15]. The perfomance of S-ALOHA in channel fading Nakagami with the same parameter for test packet and interfering packet as well [16]. In communication of micro diversity, the rate of fading parameter for main signal and interfering signal is not always the same. In this research we will analyze the throughput of CDMA S-ALOHA in Nakagami/Nakagami fading channel with different parameter of fading, CDMA S-ALOHA system utilize capture effect. Capture effect is added in system intended to overcome the decreasing of effective throughput because the amount of bits in transmitted packets is decreasing, so capture effect is used to overcome this problem because in S-ALOHA techniques, the receiver in BS can capture a packet successfully as many packets transmitted together in one slot. Thus, effective throughput depend on capture probability and probability of succeed packet that transmitted. Therefore, it is very interesting to analyze throughput of CDMA S-ALOHA in Nakagami/Nakagami fading channel in a different parameter of fading in capture effect. The perfomance in a communication system that use random access method can be measure through throughput parameter [17],[18]. 
The contribution in this research is analysis the throughput of CDMA S-ALOHA that use BER with different dual model path gain in model Nakagami/Nakagami fading channel with capture effect. We analyze the throughput through calculation in a different dual model path gain for main signal or interfering signal. To enhance throughput of CDMA S-ALOHA, we propose use capture delay [19]-[21]. Capture delay is potential to make a packet can receive well as there is another packets in the same slot so the throughput is getting improvement.

\section{Model System}

We can use system observe in figure 4.1. Signal from transmitter $(T X), S$, transmitted through in a channel to receiver $(R x)$. In $\mathrm{Rx}$, the received signal consist of main signal, $X$, and $(L-1)$ multipath random signal, $Y$, that is interfering signal. The combined between $X$ and $Y$ that receive from $R X$ is always assumed have the same model. In micro diversity, model path gain between signal $X$ and $Y$ might be different.

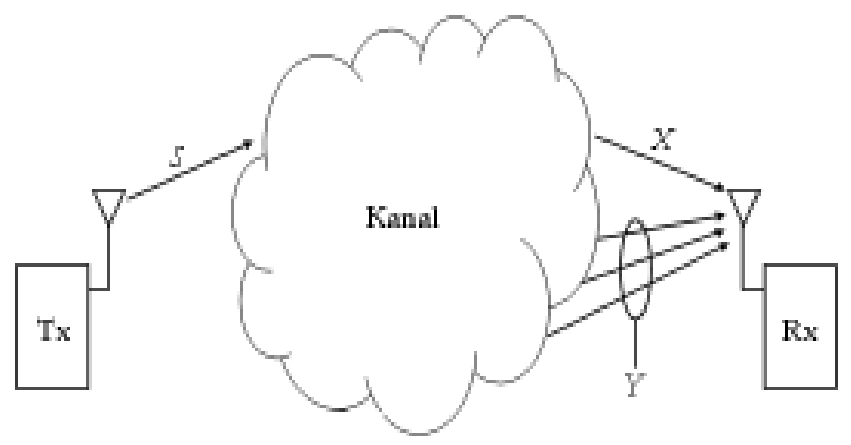

Figure 4.1 Model system

\subsection{Model Transmitter}

Transmitted signal by user $k^{\text {th }}$ is given by [8]:

$$
S_{k}(t)=A a_{k}(t) b_{k}(t) \cos \left(\omega_{c} t+\theta_{k}\right)
$$

$A$ is amplitude of signal where $A=\sqrt{2 P}$ with $\mathrm{P}$ is signal power [3]-[7], is carrier frequency, and $\theta_{k}$ is signal phase $k^{\text {th }}$. Signal of spread spectrum code (SSC) $a_{k}(t)$ from $k^{\text {th }}$ is used for spreading process that is an order of periodic signal with amplitude is one (unit amplitude), there are positive and negative in a square pulse with duration Tc [1], and can state as

$a_{k}(t)=\sum_{i=-\infty}^{\infty} a_{i}^{k} P_{T_{c}}\left(t-i T_{c}\right)$ 
$a_{i}^{k}$ is chip amplitude $i^{\text {th }}, P_{T_{c}}(t)=1$ for $0 \leq t \leq T$ and $P_{T_{c}}(t)=0$ and another $t$. Data signal from user $k^{\text {th }}$ is an order of periodic signal with amplitude is one (unit amplitude), there is positive and negative in square pulse with duration $\mathrm{T}$, and can be state as

$b_{k}(t)=\sum_{j=-\infty}^{\infty} b_{j}^{k} P_{T}(t-j T)$

$b_{j}^{k}$ is amplitude of symbol data at time slot $j^{\text {st }}$ and $k=1,2, \ldots, K$, and $K$ is the amount of active users simultaneously. Duration of data signal is $T=N T_{c}, N$ is length of PN code.

\subsection{Model Channel}

If there is L-path signal that transmit in a channel, there is one desired signal at least in a channel and $L-1$ multipath fading signal. Impulse response from low passer (lowpass equivalent impulse response) pass band channel for the link between transmitter $k^{\text {th }}$ and receiver is given [8]:

$h_{k}(\tau)=\sum_{l=1} \beta_{l k} \delta\left(\tau-\tau_{l k}\right) e^{j \Phi_{l k}}$

$\beta_{l k}$ is path gain $l^{\text {th }}, \Phi_{l k}$ is path phase $l^{\text {th }}$ that assumed uniformly in $[0,2 \pi]$, and $\tau_{l k}$ is path time delay that assumed uniformly in $[0, T]$, where $I=1,2, \ldots, L$ is the amount of path.

Received signal from receiver is given by [8]:

$r(t)=\operatorname{Re}\left\{\sum_{k=1}^{K} \int_{-\infty}^{\infty} h_{k}(\tau) \tilde{S}_{k}(t-\tau) \exp \left(j \omega_{c} t\right) d \tau\right\}+n(t)$

$\tilde{S}_{k}(t)$ is complex envelope from $S_{k}(t)$ for $\theta_{k}=0$ and $\operatorname{Re}\{$. $\}$ state as real part of the complex number. Substitutes equation ( 1 ) and (4) to equation (5), so we get [8]:

$r(t)=A \sum_{l=1}^{L}\left\{\beta_{l 1} a_{1}\left(t-\tau_{l 1}\right) \times b_{1}\left(t-\tau_{l 1}\right) \times \cos \left(\omega_{c} t+\Phi_{l 1}\right)\right\}+$

$A \sum_{l=1}^{L} \sum_{k=2}^{K}\left\{\beta_{l k} a_{6)^{1}}\left(t-\tau_{l k}\right) \times b_{k}\left(t-\tau_{l k}\right) \times \cos \left(\omega_{c} t+\Phi_{l k}\right)\right\}+n(t)$

Where $n(t)$ is white Gaussian noise with power spectral density (psd) worth of $N_{0} / 2$ (W/ $\mathrm{Hz}$ ), is the amplitude of fading with Nakagami distribution. In (6), desired users is prefer to first user $(k=1)$, while second user until user are assumed as interfering signal. 


\subsection{Model Receiver}

If assumed that delay and phase from receiver is synchronize with first user so decission statistic to detect signal from that user is[8]:

$\xi_{j}=\int_{0}^{T} r(t) a_{1}\left(t-\tau_{j 1}\right) \cos \left(\omega_{c} t+\Phi_{j 1}\right) d t$

Substitute (6) to (7), therefore [8]:

$\xi_{j}=\beta_{j 1} \frac{A T}{2} b_{0}^{1}+$

$\frac{A}{2} \sum_{i=1, i \neq j}^{L}\left\{\begin{array}{l}\beta_{l 1} \cos \left(\Phi_{l 1}-\Phi_{j 1}\right) \\ \times\left[b_{-1}^{1} R_{11}\left(t_{i 1}\right)+b_{0}^{1} \hat{R}_{11}\left(t_{i 1}\right)\right]\end{array}\right\}+$

$\frac{A}{2} \sum_{k=2}^{K} \sum_{i=1}^{L}\left\{\begin{array}{l}\beta_{l k} \cos \left(\Phi_{l k}-\Phi_{j k}\right) \\ \times\left[b_{-1}^{k} R_{k 1}\left(t_{i k}\right)+b_{0}^{k} \hat{R}_{k 1}\left(t_{l k}\right)\right]\end{array}\right\}+v$

Where and are an order data bits from signal of user , , and $v$ is Gaussian with zero mean and variance $N_{0} T / 4$. First row in (8) represent desired signal to detect. Second row is $(L-1)$ self-interference that desired (first user) effected by side lobe function of autocorrelation of SSC from first user. Third row is $L(K-1)$ multi-user interference or MAI from $(K-1)$ different active user simultaneously in a system. The last row is Gaussian RV affected by AWGN. If diversity technique that used is MRC with M order. So we can define a random variable (random variable - RV), in [10]:

$$
\alpha=\sum_{m=1}^{M} \beta_{m}^{2}
$$

Where $\beta_{m}$ is path gain in (8).

\section{Bit Error Rate of CDMA}

The calculation of BER's CDMA can use through SIR and PDF. Calculation BER intends to model of Nakagami fading channel, it means that desired signal and interference signal are modeled in Nakagami model. While packet gain that use for desired signal is assumed has different rate with path gain for interfering signal. 
3.1. Signal to Interference Ratio (SIR)

We can simplify equation (8), to [10]

$$
\begin{aligned}
& \xi_{j}=\beta_{j 1} \frac{A T}{2} b_{0}^{1}+ \\
& \frac{A}{2} \sum_{l=1, l \neq j}^{L} \beta_{l 1} \cos \left(\Phi_{l 1}-\Phi_{j 1}\right) W_{l 1}+ \\
& \frac{A}{2} \sum_{k=2}^{K} \sum_{l=1}^{L} \beta_{l k} \cos \left(\Phi_{l k}-\Phi_{j k}\right) W_{l k}+v
\end{aligned}
$$

with $W_{l k}=\left[b_{-1}^{k} R_{k 1}\left(t_{l k}\right)+b_{0}^{k} \hat{R}_{k 1}\left(t_{l k}\right)\right]$. First row is desired signal that has avarage power [8],[10]

$$
P_{\text {signal }}=\left(\frac{A T}{2}\right)^{2} \beta_{j 1}^{2}
$$

constant. While second row, third and fourth have self interefence, MAI, and noise with a given power is [10]

$$
\begin{aligned}
& P_{\text {int } 2}=\frac{A^{2}}{4} \sum_{l=1, l \neq j}^{L} E\left[\left(\beta_{l 1} \cos \left(\Phi_{l 1}-\Phi_{j 1}\right)\right)^{2}\right] E\left[W_{l 1}^{2}\right] \\
& P_{\text {int 3 }}=\frac{A^{2}}{4} \sum_{k=2}^{K} \sum_{l=1, l \neq j}^{L} E\left[\left(\beta_{l k} \cos \left(\Phi_{l k}-\Phi_{j 1}\right)\right)^{2}\right] E\left[W_{l k}^{2}\right]
\end{aligned}
$$

$$
P_{\text {noise }}=\frac{N_{0} T}{4}
$$

Where $=2 T^{2} / 3 \mathrm{~N}$ for the system that use Gold code [10]. SIR is defined as the comparison of main signal's power to sum of total interfering signal's power and noise can be state as [10]

$$
S I R=\frac{1}{2} \frac{\mathrm{P}_{\text {signal }}}{\left(\mathrm{P}_{\text {int } 2}+\mathrm{P}_{\text {int } 3}+\mathrm{P}_{\text {noise }}\right)}
$$


Substitute (11) to (14) and (15) to get result [10]

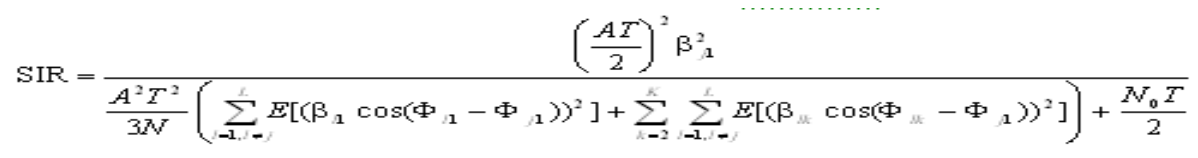

Equation (15a) can state in another form as [10]:

$$
\mathrm{SIR}=\frac{\alpha E_{z} / N_{0}}{\mathbf{1}+\left[(L-\mathbf{1}) \frac{E_{b}}{N_{0}} E\left[\boldsymbol{\beta}_{l 1}^{2}\right] \frac{2}{3 N}\right]+\left[L(K-\mathbf{1}) \frac{E_{b}}{N_{0}} E\left[\boldsymbol{\beta}_{l k}^{2}\right] \frac{2}{3 N}\right]}
$$

$\alpha_{m}$ given by (9). If $E\left[\beta_{l 1}^{2}\right]=\Omega_{x}$ and $E\left[\beta_{l k}^{2}\right]=\Omega_{y}$,

$\Omega_{x}$ is power signal of component in self interference and

$\Omega_{y}$ is power signal of component of MAI with $\alpha H$ is

SIR, thus equation ( 16 ) can be written as

$$
\mathrm{SIR}=\frac{\alpha E_{b} / N_{0}}{1+\frac{E_{b}}{N_{0}} \frac{2}{3 N}\left[(L-1) \Omega_{x}\right]+\left[L(K-1) \Omega_{y}\right]}=\alpha H
$$

\subsection{Probability Density Function Signal in Nakagami Channel Fading}

If $X$ is RV that state as transmitted power signal and go through distributed fading of Nakagami, thus $X$ will have pdf as follows[2]:

$$
P_{X}(x)=\left(\frac{m_{x}}{\Omega_{x}}\right)^{m_{x}} \frac{x^{m_{x}-1}}{\Gamma\left(m_{x}\right)} \exp \left(-\frac{m_{x} x}{\Omega_{x}}\right)
$$

$X$ is instantaneous power that goes through fading; $m_{x}$ is parameter of Nakagami fading.

If $Y$ is RV that state power from $L$ signal that go through distributed fading of Nakagami, thus $Y$ will have pdf as [2]:

$$
p_{Y}(y)=\left(\frac{m_{y}}{\Omega_{y}}\right)^{m_{y} L} \frac{y^{m_{y} L-1}}{\Gamma\left(m_{y} L\right)} \exp \left(-\frac{m_{y} y}{\Omega_{y}}\right)
$$

$y$ is power from instantaneous signal that go through fading, $m_{y}$ is parameter fading of Nakagami. To calculate BER with two kind of model path gain, thus can defined as new $\mathrm{RV}$, that is, with each of $X$ and $Y$ have given pdf from (18), (19). Pdf from $A$ calculate in: 
$p_{\mathrm{A}}(\alpha)=\int_{-\infty}^{\infty} p_{X}(x) p_{Y}(y) d y=\int_{-\infty}^{\infty} p_{X}(\alpha-y) p_{Y}(y) d y$

The calculation of combined pdf from signal $X$ with distributed Nakagami and signal $Y$ with distributed Nakagami can obtain thorough subtitute equation (18) and (19) to (20) as follows:

$$
\begin{aligned}
& p_{\alpha}(\alpha)=\int_{0}^{\alpha}\left(\frac{m_{x}}{\Omega_{x}}\right)^{m_{x}} \frac{(\alpha-y)^{m_{i}-1}}{\Gamma\left(m_{x}\right)} \exp \left(-\frac{m_{x}(\alpha-y)}{\Omega_{x}}\right) \times\left(\frac{m_{y}}{\Omega_{y}}\right)^{m, z} \frac{y^{m^{\prime} z-1}}{\Gamma\left(m_{y} L\right)} \exp \left(-\frac{m_{y} y}{\Omega_{y}}\right) d \alpha \\
& =\frac{\left(\frac{m_{x}}{\Omega_{x}}\right)^{m_{x}}\left(\frac{m_{y}}{\Omega_{y}}\right)^{m^{\prime} L}}{\Gamma\left(m_{x}+m_{y} L\right)} \exp \left(-\frac{m_{x} \alpha}{\Omega_{x}}\right) \alpha^{m^{+}+m^{\prime} L-1} \times_{1} F_{1}\left[m_{y} L, m_{x}+m_{y} L, \alpha\left(\left(\frac{m_{x}}{\Omega_{x}}\right)-\left(\frac{m_{y}}{\Omega_{y}}\right)\right)\right]
\end{aligned}
$$

\subsection{Bit Error Rate}

Conditional of BER CDMA in modulated system of noncoherent BPSK determined by [2],[9]:

$P_{e}(\alpha)=\frac{1}{2} \exp (-\alpha H)$

is given SIR by equation(17).

BER from CDMA system can calculated in equation [2],[9]:

$$
P_{e}=\int_{0}^{\infty} P_{e}(\alpha) p_{\mathrm{A}}(\alpha) d \alpha
$$

is pdf from $A$ that given by (21).

BER CDMA with dual model path gain in the Nakagami/Nakagami fading channel obtained through substitute equation (21) and (22) to (23) that is:

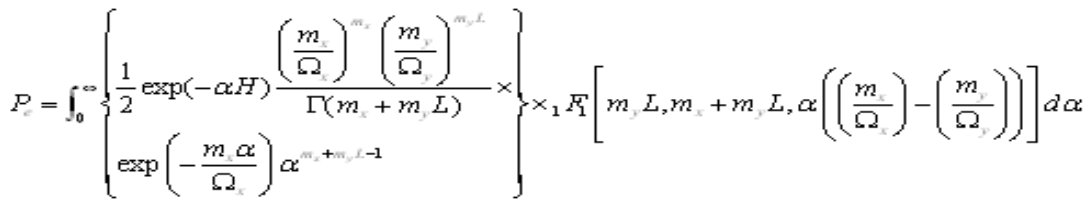

After calculation we get

$$
\begin{aligned}
& P_{e}=\frac{1}{2}\left(\frac{m_{x}}{\Omega_{x}}\right)^{m_{x}}\left(\frac{m_{y}}{\Omega_{y}}\right)^{m_{x} I}\left(H+\frac{m_{x}}{\Omega_{x}}\right)^{-m_{x}-m_{x} I}\left(\mathbf{1}-\frac{\left(\frac{m_{x}}{\Omega_{x}}\right)-\left(\frac{m_{y}}{\Omega_{y}}\right)}{\left(H+\frac{m_{x}}{\Omega_{x}}\right)}\right)^{-m_{y} I} \\
& \text { If } q=\Omega_{x} / \Omega_{y} \text {, thus } \\
& P_{e}=\frac{1}{2}\left(\frac{m_{x}}{q}\right)^{m_{x}} m_{y} m_{y} I\left(H+\frac{m_{x}}{q}\right)^{-m_{x}-m_{y} I}\left(1-\frac{\left(\frac{m_{x}}{q}\right)-m_{y}}{\left(H+\frac{m_{x}}{q}\right)}\right)^{-m_{y} I}
\end{aligned}
$$


is gamma function, is, is the power of signal component self interference, is the power of component signal of MAI, is the amount of active user simultaneously. Nakagami distribution can used as Rayleigh distribution as.

\section{Capture Probability}

In cellular communication where there is different variety of signal power between one and another signal as effected by MS motion, effect fading, and variety of distant between transmitter and receiver. These things make possible that a packet can receive well as there are another packets in a same slot. This phenomenon is called capture effect. These phenomenons escalate throughput system.

\subsection{Delay Capture}

In this research, we will explain about delay capture, where capture probability from delay capture effect is given by [19],[20],[21]:

$$
C_{n}=\left\{\begin{array}{cc}
0 \Rightarrow & k=0 \\
1 \Rightarrow & k=1 \\
(1-Q)^{k} \Rightarrow & k \geq 2
\end{array}\right.
$$

$\mathrm{Q}$ is ratio of delay capture that is comparison between capture time and different time of maximum arriving packet. For perfect capture effect, and without capture effect.

\section{Throughput of CDMA S-ALOHA}

Throughput of CDMA S-ALOHA in the channel fading we state as [15],[16],[20]-[25] :

$$
S=G\left(\sum_{k=1}^{\infty} P_{k}(k)\right) \cdot\left(1-P_{e}\right)^{L_{B}}
$$

Where is the lenght of a packet, is probability frame that happen along period of frame in S-ALOHA that use Poisson distribution:

$$
P_{k}(k)=\frac{G^{k} e^{-G}}{k !}
$$

Throughput of CDMA S-ALOHA in channel fading apply delay capture effect

$$
S=G\left(\sum_{k=\mathbf{1}}^{\infty} P_{k}(k)\right) \cdot\left(\mathbf{1}-P_{e}\right)^{L_{B}} \cdot C_{k}
$$




\section{Results and Analysis}

Table 1. Used Parameter

\begin{tabular}{|l|l|}
\hline Parameter & Rate \\
\hline The amount of signal from signal & $n=3$ \\
\hline The amount of total path & $L=5$ \\
\hline The amount of users & $K=20$ \\
\hline Processing gain & $N=15,31,63$ \\
\hline Average power of main signal & $\Omega_{x}=5$ \\
\hline Average power of MAI signal & $\Omega_{y}=5$ \\
\hline $\begin{array}{l}\text { Parameter of Nakagami fading from } \\
\text { main signal }\end{array}$ & $m_{x}=3$ \\
\hline $\begin{array}{l}\text { Parameter of Nakagami fading from } \\
\text { interference power signal }\end{array}$ & $m_{y}=3$ \\
\hline Signal to noise ratio & $\frac{E_{b}}{N_{0}}=30$ \\
\hline Capture ratio & $Q=0.01$ \\
\hline The length of packet & $L_{b}=500$ \\
\hline Offered load & $G=0-40$ \\
\hline
\end{tabular}

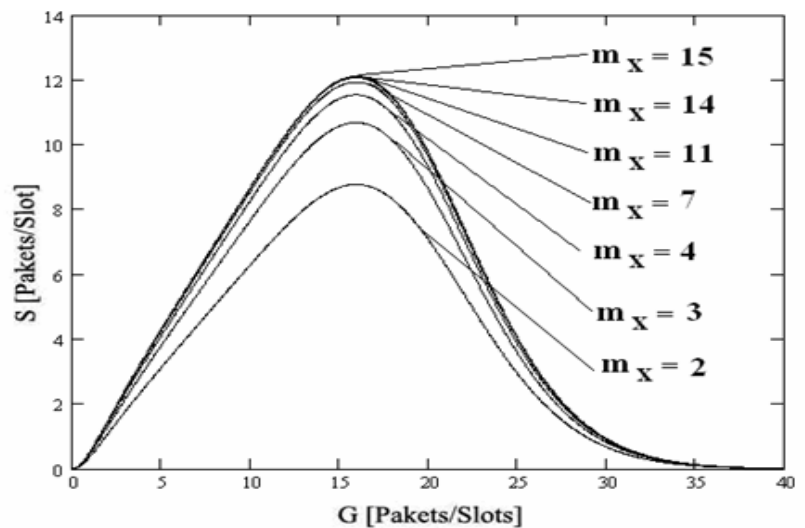

Figure 1. Throughput of CDMA S-ALOHAin the Nakagami/Nakagami fading channel with capture effect with rate of changed and parameter rate of.

Figure 1. Shows that throughput of CDMA S-ALOHA in the Nakagami/Nakagami fading channel with capture effect and rate of will change as parameter rate of. Figurel 
obtain from equation (30). Figure 1 shows that the increasing of effect the throughput of S-ALOHA is increase also, this thing occur because the increasing of parameter rate of , of course the increasing of main power signal higher than the power of interfering signal.

\section{Conclusions}

We can observe that the throughput of CDMA S-ALOHA will getting improvement if the rate of is higher, lenght of code and capture effect. Throughput of CDMA S-ALOHA is worsen if the amount of users is increasing, capture ratio.

\section{References}

[1] M. B. Pursley (1977). "Performance evaluation for phase-coded spread-spectrum multiple-access communication--Part I: System Analysis," IEEE Trans. Comm., vol COM-25, hal. 795-799

[2] V. A. Aalo dan J. Zhang (1999) "On the effect of cochannel interference on average error rates in nakagami-fading channels, "IEEE Comm. Lett., vol. 3, no.5, hal. 136-138.

[3] R. K. Morrow, J r. dan J. S. Lehnert (1989). "Bit-to-bit error dependence in slotted DS/SSMA packet systems with random signature sequences," IEEE Trans. Comm., vol. 37, no. 10, hal. 1052-1061.

[4] J. M. Holtzman (1992). "A simple, accurate method to calculate spread-spectrum multiple-access error probabilities," IEEE Trans. Comm., vol. 40, no. 3, hal. 461464

[5] J. M. Holtzman (1992). "On calculating DS/SSMA error probabilities," IEEE $2^{\text {nd }}$ International Symposium on Spread Spectrum Techniques and Applications, Jepang, Nopember.

[6] M. O. Sunay dan P. J. McLane (1996). "Calculating error probabilities for DS CDMA systems: when not to use the gaussian approximation," IEEE Globecom 96, vol. 3, hal. 1744-1749.

[7] J. Cheng dan N. C. Beaulieu (2002). "Accurate DS-CDMA bit-error probability calculation in rayleigh fading," I EEE Trans. Wire. Comm., vol. 1, no. 1, hal. 3-15. 
[8] M. Kavehrad dan P. J. McLane (1985). "Performance of low-complexity channel coding and diversity for spread spectrum in indoor, wireless communication," AT\&T Tech. Journal, vol. 64, no. 8, hal. 1927-1965.

[9] C. Chayawan dan V. A. Aalo (2003). "Average symbol error rate of maximal ratio combining scheme in the presence of multiple cochannel interferers," ECTI Trans. Electrical Eng., Electronics, and Comm., vol. 1, no. 1, hal. 38-45.

[10] C. Lee dan Y. J eon (1998). "BER analysis of asynchronous DS-CDMA over a rician fading channel, "IEICE Trans. Fund., vol. E81-A, no. 7, hal. 1479-1482.

[11] F. Sasamori dan F. Takahata ( 1999). "Theoretical and approximate derivation of bit error rate in DS-CDMA systems under rician fading environment," IEICE Trans. Fund., vol. E82-A, no. 12, hal. 2660-2668.

[12] Y-D. Yao dan A. U. H. Sheikh (19920. "I nvestigation into cochannel interference in microcellular mobile radio systems," IEEE Trans. Vehic. Tech, vol. 41, no. 2, hal. 114-123.

[13]. A. Sheikh, Y-D Yao dan X. WU (1990). "The ALOHA system in shadowed mobile radio channel with slow or fast fading", IEEE Transactions on Vehicular Technology, Vol.39, No.4, pp.289-286.

[14]. J. A. Roberts dan T. J Healy (1980). "Packet radio performance over slow rayleigh fading channel", IEEE Transaction on Vehicular Technology, Vol. COM-28, No.2, pp.279-286.

[15]. J. C. Anbark dan W. Van Blitterswijk (1987). "Capacity of slotted ALOHA in rayleigh fading channel", IEEE Journal on Selected Areas in Communication, Vol. SAC-5, No.2, pp.261-265.

[16]. Sand A. Al-Semari dan Mohsen Guizani (1997). "Channel throughput of slotted ALOHA in nakagami fading environtment", IEEE Transaction on Communications.

[17] A. Ogawa, T. Yamazato, M. Katayama and Jamalipour (1998) "Introduction to CDMA ALOHA", IEEE GLOBECOM'98, TU13. 
[18] Tseng, Shu-Ming (2003). "A high-throughput multicarrier DS CDMA / ALOHA network", IEICE Trans.Commun, Vol.E86-B.

[19]. K. Cheun and S. Kim (1998)." J oint delay-power capture in spread-spectrum packet radio networks," IEEE Trans, Commun,. Vol.46, No. 4, pp 450-453.

[20]. M. S. Do, Y. J. Park, J. Y. Lee," Capture with delay and power randomization in spread-spectrum CDMA slotted ALOHA system," IEEE Trans, Commun, vol, 26, July 20, pp 996-1006.

[21]. M. S. Do, Y. J. Park (2002. 2005). J. Y. Lee," The effect of spreading gain control on a CDMA slotted ALOHA System," IEEE Trans, Computer Commun, Vol, 26, pp 996-1006.

[22] Damar Wijaya dan Gunawan Wibisono (2005). "Analisa BER CDMA dengan Dua Model Path Gain Kanal Fading Nakagami," Proc. The 6 th Seminar on Intellegent Technology and Its Applications (SITIA 2005), Institut Teknologi Sepuluh November, Surabaya.

[23] Gunawan Wibisono dan Andy Prakoso (2006). "Analisis Kinerja CDMA Dengan Differensial MRC Pada Dua Model Fading,"Proc. EECCIS 2006, Brawijaya University Malang, Part 1, C-135.

[24] Hoga Saragih dan Gunawan Wibisono (2005). "Analysis Throughput Slotted ALOHA DS-CDMA in Nakagami/Nakagami Fading Channel with Capture Effect", International Conference On information and Communication technology (ICCT-UMB 2005), Universitas Mercubuana, 9-10 juni 2005, Jakarta, Hal 257-266.

[25] Hoga Saragih, Gunawan Wibisono dan Eko Tjipto Rahardjo (2006). "Analysis of Throughput S-ALOHA CDMA with Differential MRC at Two Fading Models", RPCES 2006, Universiti Teknologi Malaysia, Skundai, J ohor Bahru, 26-27 J uly 2006, Hal 275-280. 